\title{
Evaluación de Desempeño de la Función Académica: Análisis de un Sistema en el Contexto Universitario Chileno
}

\author{
Víctor E. Cancino ${ }^{(1)^{*}}$ y Tamara S. Márquez ${ }^{(2)}$ \\ (1) Universidad de Talca, Instituto de Investigación y Desarrollo Educacional, Av. Lircay s/n, Talca-Chile. \\ (e-mail: vcancino@utalca.cl) \\ (2) Universidad Santo Tomás, Sede Talca. Av. Carlos Schorr 255, Talca-Chile. \\ (e-mail: tmarquezlemp@gmail.com) \\ * Autor a quien debe ser dirigida la correspondencia.
}

Recibido Sep. 12, 2014; Aceptado Nov. 10, 2014; Versión final recibida Ene. 29, 2015

\begin{abstract}
Resumen
Este trabajo hace una revisión de elementos teóricos y empíricos sobre la evaluación desempeño desde un enfoque estratégico de la gestión de recursos humanos. El artículo analiza el sistema de evaluación de desempeño académico de una universidad estatal chilena, bajo una metodología de tipo exploratoria cualitativa basada en cinco entrevistas en profundidad a académicos de diferentes jerarquías que han ocupado cargos claves. Entre los resultados destaca el amplio conocimiento que tienen los entrevistados sobre las principales características y componentes del sistema, lo que permite contar con un diagnóstico inicial sobre las fortalezas y debilidades que presenta. A modo de conclusión, se plantea la alta valoración que expresan los entrevistados sobre la importancia estratégica del sistema de evaluación para la universidad. Ellos hacen hincapié en la necesidad de implementar algunas mejoras de naturaleza táctica y operativa para fortalecer su eficacia y eficiencia, contribuyendo en mejor forma al desarrollo de un proyecto universitario sustentable.
\end{abstract}

Palabras clave: gestión académica, desempeño académico, docencia universitaria, evaluación de desempeño

\section{Performance Assessment of Academic Function: Analysis of a System in the Chilean University Context}

\begin{abstract}
This work reviews theoretical and empirical elements regarding performance assessment, from a strategic point of view within a human resources management approach. The article analyzes the performance assessment system of a Chilean public university, under a qualitative exploratory methodology based on five in-depth interviews to faculty members across different academic hierarchies that have worked in key university roles. Results emphasize the vast knowledge these the professors have regarding the main characteristics and components of the system, which allows to diagnose strengths and weaknesses of it. As a conclusion, the high relevance the interviewees place on the performance assessment system is presented. They emphasized on the need of implementing some tactical and operative improvements to improve efficacy and efficiency of the system, to contribute in a better way to a sustainable university project.
\end{abstract}

Keywords: academic management, academic performance, university teaching, performance evaluation 


\section{INTRODUCCION}

En la actualidad, la gestión de recursos humanos destaca la relevancia estratégica del desarrollo del talento y la productividad de las personas para el logro de objetivos organizacionales (Bohlander, Snell, y Sherman, 2001), así como su vinculación con la estrategia corporativa (Hatum, 2009). Se destaca también al recurso humano como fundamental para el desarrollo de toda organización, siendo uno de los principales factores que actúan como fuente de competencias centrales para alcanzar una ventaja competitiva sostenible (Hitt, Ireland y Hoskisson, 2004). En este contexto, es fundamental para toda organización contar con un sistema de evaluación de desempeño, proceso entendido como la "valoración, sistemática, de la actuación de cada persona en función de las actividades que desempeña, las metas y los resultados que debe alcanzar, las competencias que ofrece y su potencial desarrollo" siendo también "un potente medio para resolver problemas de desempeño y para mejorar la calidad de trabajo y la calidad de vida de las organizaciones" (Chiavenato, 2009). El proceso de evaluación de desempeño considera que las personas son evaluadas en un contexto laboral y en un puesto de trabajo específico, cuyo análisis situacional dependerá de: i) las funciones que se le hayan atribuido; ii) de las condiciones laborales del puesto de trabajo; y iii) de las características personales de cada trabajador (Chiavenato, 2000).

Evaluar el desempeño de las personas en una organización es una herramienta útil para alcanzar los objetivos organizacionales, facilitando la mejora continua en los procesos y tareas, pudiendo tener un alto impacto en función del tipo de sistema de evaluación que se utilice. Hatum (2009) señala que de acuerdo a la manera en que se evalúe (qué, cómo y cuándo), serán los resultados que se obtengan a nivel de desempeño institucional y la articulación que se logre con los objetivos estratégicos de una organización. Desarrollar un sistema de evaluación de desempeño, implica abocarse a superar las limitaciones y debilidades que pueda tener el proceso de evaluación, para evitar que este pierda valor frente a las personas y la organización, siendo necesario utilizar medios para disminuir estos efectos indeseables (Chiavenato, 2009).

Para ello, es posible utilizar algunos enfoques más pertinentes al momento de evaluar, como la evaluación por competencias y/o la evaluación en $360^{\circ}$, siendo fundamental seleccionar un método de evaluación de acuerdo a las características de la organización, así como el entrenamiento adecuado de los evaluadores de manera que no caigan en conductas que perjudiquen el proceso. La evaluación en $360^{\circ}$ es definida por Chiavenato (2009) como "...una evaluación circular de todos los elementos que tienen alguna interacción con el evaluado..." (p.265), siendo una forma más rica de evaluación porque produce información desde todas perspectivas. Este enfoque consiste en que un grupo de personas valora el desempeño de otra, a través de una serie de ítems o factores definidos previamente y que se pueden observar en el desempeño diario del sujeto. (Alles, 2005)

Por otra parte, Gil (2007) entiende la evaluación por competencias "como un proceso por el cual se recoge información acerca de las competencias desarrolladas por un individuo y se comparan éstas con el perfil de competencias requerido por un puesto de trabajo, de tal manera que pueda formularse un juicio de valor sobre el ajuste al mismo" (p.87). A diferencia de la evaluación tradicional, la evaluación por competencias permite respetar las individualidades de las personas y las características propias del puesto de trabajo, evitando caer en generalizaciones que dificultan el proceso de evaluación de desempeño, que pueden generar resultados erróneos que traerían consecuencias para las personas y la organización. Adicionalmente, señala que "la evaluación de competencias, cuando se enmarca en la evaluación del desempeño, supone una comparación entre las competencias demostradas por el individuo en su trabajo y las competencias exigidas para un desempeño exitoso en el puesto que ocupa" (Ibíd, p.92). Los enfoques señalados no son excluyentes, ya que una organización puede utilizar una evaluación en $360^{\circ}$, donde los factores evaluados sean las competencias en el grado que son requeridas para un puesto de trabajo, ambos enfoques pueden constituir un sistema integrado de evaluación de desempeño.

Finalmente, se debe considerar que los sistemas de evaluación de desempeño son absolutamente necesarios en toda institución para mejorar sus procesos y alcanzar óptimos estándares de calidad. Un sistema de evaluación de desempeño tendrá debilidades que hay que considerar, evitando que sea percibido como inequitativo o injusto, promoviendo su utilidad para mejorar el desempeño. Los sistemas de evaluación deben estar diseñados "a medida" para la organización, de manera que respondan a sus necesidades y facilite el logro de sus objetivos estratégicos.

\section{PROFESIÓN ACADÉMICA Y EVALUACIÓN DE DESEMPEÑO}

Para Salmi (2009), la educación superior cumple un rol particularmente relevante en apoyar la creación de una base sustantiva de capital humano, para contribuir a un eficaz sistema nacional de innovación, ayudando de esta forma, a que los países desarrollen economías competitivas sobre la base de recursos 
humanos calificados, la creación, aplicación y difusión de nuevos conocimientos y tecnologías. También existe un amplio consenso sobre la centralidad que tiene la educación superior en ámbitos como la formación permanente, la investigación básica y aplicada, así como en el desarrollo social y tecnológico de un país (Brunner, 2014; Vain, 1998). Altbach, Reisberg \& Rumbley (2009), plantean que los desafíos de la profesión académica son cada vez más complejos y el rol del académico-investigador se ha posicionado por sobre otras tareas relevantes, aun cuando, en sistemas académicos diferenciados, no todos los profesores se centrarán en la investigación, ya que la mayoría de los académicos principalmente enseñan, y sus cargas de trabajo así lo reflejan.

Para Macfarlane (2011), la profesión académica es compleja por las múltiples tareas que se deben realizar, ya que en la educación superior ha predominado la noción 'del académico como un 'todo terreno': alguien que enseña, investiga y realiza una variedad de servicios o tareas administrativas, como líder de un departamento o de trabajo como asesor de los estudiantes"(p.60). Cavalli y Moscati (2010) indican que hace un par de décadas atrás los académicos contaban con condiciones de trabajo más ventajosas (baja evaluación, alta autonomía y libertad); pero actualmente enfrentan alta competencia, evaluación y rendición de cuentas. Junto con ello, se observan cambios en las condiciones laborales, por una parte "la disminución de las oportunidades de trabajo a tiempo completo (debido al aumento de la universidad del sector privado, entre otros) ha aumentado la competencia" (p.51), así mismo, Macfarlane (2011) señala que existe una creciente tendencia a subcontratar personal para alguna de las tareas específicas del área académica, lo que genera consecuencias y diferencias en la forma en que se enfrenta la vida académica actual, "si bien existe una clara diferencia en el prestigio académico entre, por ejemplo, un docente y un profesor investigador, ambos son profesionales especializados, en vez de 'todo terreno" (p.61)

Según Bernasconi (2010, citando a Jencks y Riesman, 1968, y Clark, 1997), la profesión académica se vincula a los procesos que los autores llamaron la "profesionalización de la academia, es decir, la transformación del profesor en un experto especialista, consagrado al avance de su disciplina en el contexto de su afiliación institucional, entrenado en investigación, con dedicación completa a la actividad académica, y que genera conocimiento según estándares de desempeño establecidos y controlados por sus pares." (p.142).

Además, Bernasconi (2008) señala que en las universidades pertenecientes al Consejo de Rectores $(\mathrm{CRUCH})$ de Chile, la academia amateur ha quedado atrás dando paso a la academia profesional. Sin embargo, aún en muchas universidades privadas se continúa trabajando con profesionales dedicados exclusivamente a la docencia, o compartiendo su tiempo entre docencia y práctica; y se realiza muy poco 0 nada de investigación.

Para Berrios (2008a), la organización de la carrera académica en el sistema universitario chileno, opera sobre la base de la cultura institucional universitaria imperante, con componentes estratégicos y operativos, donde destaca la "idealización del modelo investigador"(p.49), y la existencia de diversos modelos de carrera académica que organizan esta actividad, siendo posible encontrar un patrón similar para ordenar, clasificar, categorizar y jerarquizar a los académicos en las universidades privadas y públicas, bajo los patrones que estas últimas imponen. Así mismo, según Berrios (2008b), las instituciones cuentan con diferentes categorías que "llevan asociadas exigencias diferenciadas en la evaluación del desempeño y para la promoción de sus académicos" (p.44).

La estructuración de la profesión académica en el contexto de un sistema universitario, hacen imposible pensar hoy, en una autonomía sin trabas y sin evaluación del trabajo académico, por el contrario, la rendición de cuentas y la evaluación se pueden hacer de manera que sea apropiada para el trabajo académico y no con fines punitivos (Altbach et al., 2009). El personal académico es de especial relevancia, considerando la importancia estratégica que significa para una universidad contar con recursos humanos altamente calificados que puedan alcanzar óptimos niveles de productividad en los diversos ámbitos vinculados al quehacer universitario, sin embargo, la evaluación de desempeño se ha vinculado mayoritariamente con la función docente, dejando de lado otros aspectos relevantes de la actividad académica (CINDA, 2008).

Para Fernández Lamarra y Coppola (2010, citando a Stronge, 1997; Vain, 1998 y Rueda, 2000), la evaluación de la docencia universitaria se relaciona con distintas concepciones sobre esta tarea: i) como un componente de evaluación institucional, visualizada por las instituciones con el fin de mejorar su calidad y, en general, relacionada con los procesos de evaluación interna y externa; ii) como un aspecto de las políticas institucionales para el desarrollo de los recursos humanos; iii) como una actividad cotidiana de la enseñanza, para contribuir a mejorar dicho proceso. Así también, existen experiencias que muestran la factibilidad de desarrollar sistemas que permitan evaluar competencias docentes a partir de enfoques contextualizados (Marin, Guzman, Márquez y Pena, 2013). Dada su complejidad y magnitud, la docencia 
universitaria requiere ser asumida con un alto grado de profesionalización, existiendo muchas condicionantes relacionadas a su gestión como: el tipo, tamaño y localización de la institución, la modalidad o nivel en que se imparte la docencia; siendo posible identificar al menos cinco ámbitos en la gestión docente: la gestión del currículo, la gestión de asuntos estudiantiles, la gestión del personal docente, la gestión de los recursos materiales y de información, y la planificación y la evaluación global de la función docente (Espinoza y González, 2009).

Los mecanismos utilizados para la evaluación del desempeño de los académicos son diversos, y en el caso de México se vinculan desde los 90' a la implementación de otros instrumentos de gestión de naturaleza financiera de apoyo institucional o de tipo salarial (Rueda, 2008a), así mismo, la utilización regular de sistemas de evaluación del desempeño docente, ha permitido una especialización en esta materia, ya que ha sido incorporada "a las actividades rutinarias de las universidades y se ha habilitado y especializado un número considerable de académicos en quienes descansa la responsabilidad de diseñar y conducir esta función" (Rueda, 2008b, p.8).

Las universidades chilenas, no están ajenas a los cambios de la profesión académica y su evaluación, aspecto que se ve complejizado por el dinamismo y diversidad institucional existente, que no solo se asocia al tipo de universidades que conforman el sistema (estatales, privadas tradicionales y privadas post reforma de 1980), sino también a la diversidad que se aprecia a nivel interno de cada universidad, en cuanto a la composición de su cuerpo académico: en términos de dedicación, formación y vinculación laboral (González, Brunner y Salmi, 2013). De acuerdo a Bernasconi (2008, p.21), "...la docencia es el elemento menos dispensable en los compromisos de desempeño académico que los profesores pactan con sus jefes de unidad en cada vez más universidades del país...". Sin embargo, la docencia no es el único aspecto que se debe evaluar al medir el desempeño de un académico, ya que como se ha señalado, un docente debe ser capaz de generar y aplicar conocimiento para formar a sus estudiantes, por lo que la investigación, la extensión, la asistencia técnica y la gestión, también son labores fundamentales en el desarrollo de sus actividades.

Bernasconi (2008) también señala que el establecer estándares de desempeño profesional en torno a la investigación no necesariamente implica que los perfiles de los académicos de las universidades chilenas se ajusten a este patrón; indica además que los docentes universitarios "son instructores de tiempo completo que llenan la mayor parte de su tiempo en la enseñanza, la administración y la política interna..."(p.22). También se refiere a que estos estándares de desempeño profesionales deben ajustarse a la diversidad de oficios académicos que coexisten en las universidades, en sus palabras "...esta diversidad pone un desafío grande frente a los administradores que deben establecer reglas y políticas que rigen la carrera académica" (p.23).

Fernández (2008) indica que esta materia no ha estado ausente de las políticas educativas, ya que desde fines de los años 90, como requisito para acceder a fondos concursables del Programa de Mejoramiento de la Calidad y la Equidad de la Educación Superior (MECESUP), se implementaron en algunas universidades chilenas los compromisos de desempeño y los incentivos al trabajo académico que sirven como instrumento para apoyar la planificación estratégica.

Los compromisos individuales de desempeño son los que cada profesor contrae con su unidad académica y que deben dar cuenta del trabajo que se compromete a realizar el académico ante su jefatura y la administración de la universidad. Por lo general, los compromisos se dan en los ámbitos de docencia, investigación, extensión y administración; aunque hay universidades que incluyen otras actividades como perfeccionamiento y transferencia tecnológica. Los compromisos de desempeño son flexibles, debiendo ajustarse a la normativa y políticas de la universidad, este dinamismo es necesario por la naturaleza del trabajo académico que siempre implica diversas áreas de desempeño. Realizar un compromiso de desempeño implica que su cumplimiento se complemente con incentivos, los cuales deben orientarse hacia aquellas áreas en las que la universidad busque un mayor desarrollo. Para que un compromiso de desempeño funcione como una herramienta de planificación estratégica efectiva, debe tener una evaluación, que verifique su alcance y sancione los no cumplimientos (Fernández, 2008). Para el autor, algunas universidades realizan evaluaciones periódicas a través de comisiones en las que se utiliza el compromiso de desempeño como un insumo. Los resultados de las evaluaciones son utilizados como referencia por comisiones de categorización y promoción que jerarquizan la carrera de los académicos. Estas herramientas, cumplen dos funciones dentro de la planificación estratégica, por un lado la de socializar los objetivos de desarrollo en la organización y por otro servir de instrumento para el alcance de ellos. Por lo que claramente los compromisos de desempeño, la evaluación académica y los incentivos, deben estar alineados con los objetivos estratégicos institucionales. 
El diseño de un sistema de evaluación de desempeño, debe ser cuidadoso en el énfasis que se dé a los diferentes indicadores y criterios de evaluación, ya que esto moldeará el desempeño de las personas y la calidad de sus actividades, siendo fundamental la decisión de qué, quién(es), cómo y cuándo evaluar. La calidad del sistema y los procesos de evaluación de desempeño, conducirán a los académicos a ajustar sus expectativas y productividad, aspecto sustantivo para una organización universitaria, considerando la relevancia que tiene la evaluación de desempeño en el logro de la calidad educativa (Rocha, 2013).

\section{EVALUACIÓN ACADEMICA EN LA UNIVERSIDAD DE TALCA}

Para Rojas (2014), el desarrollo sostenido que ha tenido la Universidad de Talca se sustenta en "un conjunto de pilares fundamentales, los cuales tempranamente se fueron desarrollando con una mirada estratégica de mediano y largo plazo[..].". Es así, como a fines de la década de 90' la universidad desarrolló un acucioso proceso de planificación estratégica que involucró a toda la comunidad universitaria, lo cual se ha mantenido y perfeccionado, contando en la actualidad con un modelo de control de gestión que facilita el diseño, implementación y evaluación estratégica a nivel institucional.

La evaluación de desempeño académico en la Universidad de Talca, tiene su precursor en los compromisos de desempeño académico (desde 1996), existiendo un avance gradual que comprendió posteriormente la instalación de un sistema de calificación académica (1997-1999), contando desde 2006 con un Sistema de Compromiso y Autoevaluación del Desempeño Académico (SCADA) (Huerta y González, 2010). En el marco del actual sistema de gestión estratégica, el modelo de control de gestión facilita el proceso de convergencia entre los compromisos de desempeño de las unidades (CDUs) y los compromisos de desempeño individual de los académicos (CDAs), siendo parte sustantiva de la estructura de decisiones para los procesos de evaluación.

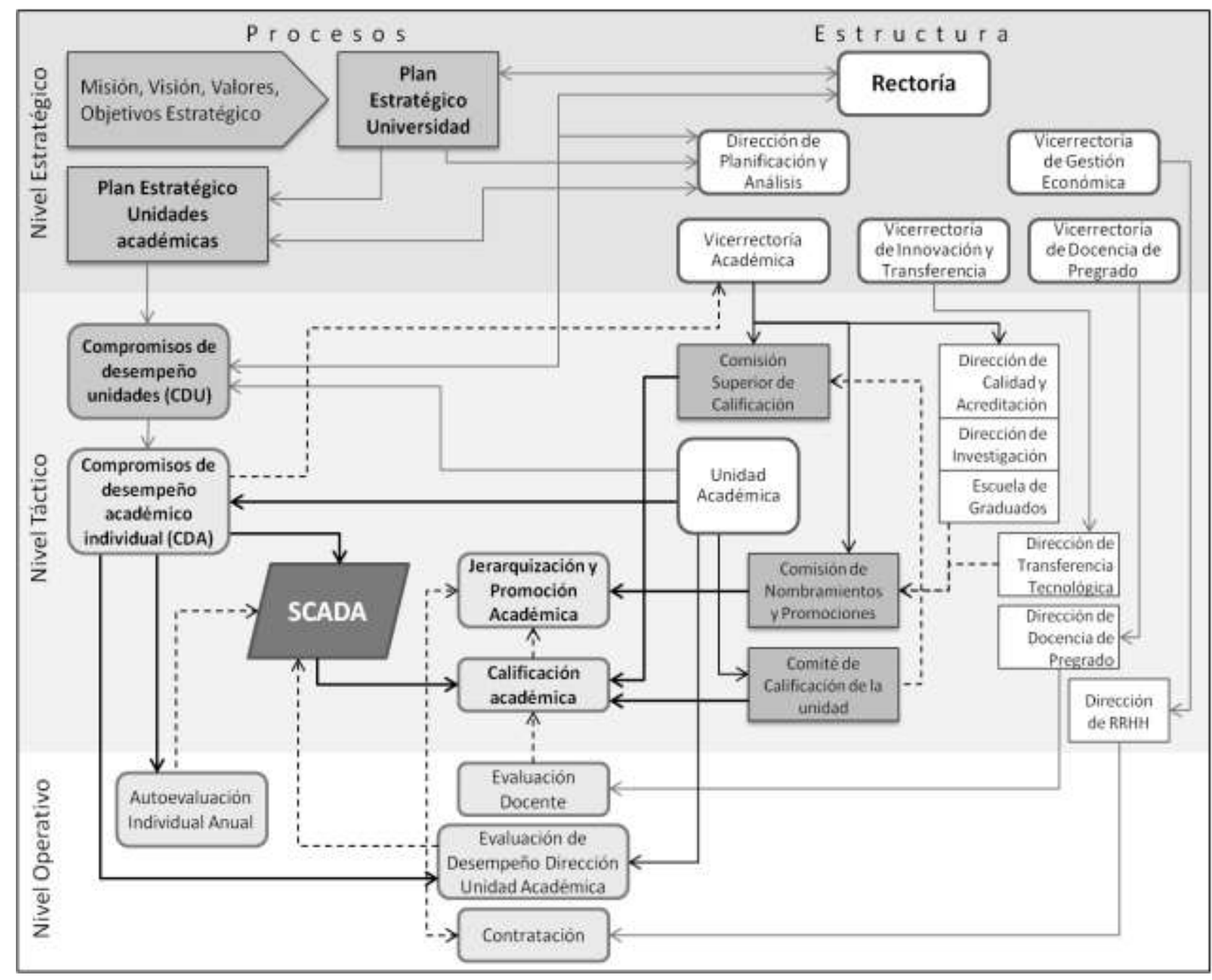

Fig. 1: Esquema de sistema de evaluación de desempeño académico de la Universidad de Talca 
El sistema de evaluación de desempeño académico consta de dos procesos independientes entre sí, por un lado se encuentra el proceso de Jerarquización y Promoción Académica, y por otro, el sistema de Calificación Académica, que se asocia directamente a un proceso de evaluación de desempeño, cuyo diseño global se muestra en la figura 1.

La Ordenanza General del Académico (UTALCA, 2013), establece que los académicos del cuerpo regular son jerarquizados en función de sus méritos en las siguientes categorías: Profesor Titular, Asociado, Asistente o Instructor. Adicionalmente, el cuerpo académico no regular, se divide en las siguientes jerarquías: Profesor Emérito, Investigador Titular, Profesor Visitante, Conferenciante, Profesor de Práctica o Asistente. Estas jerarquías tienen asociado un tipo de relación contractual, nombramientos que tienen tiempos definidos ( 1 o 2 años) o indefinido (hasta edad de retiro), con responsabilidades y obligaciones asociadas a la jerarquía que guiarán posteriormente los procesos de calificación.

La jerarquización y promoción, consiste en el proceso de análisis de antecedentes y méritos del académico, para determinar el lugar que ocupará en la jerarquía señalada anteriormente. Es un requisito ineludible para el ingreso y promoción de un académico. Si bien, todo académico está obligado a ser jerarquizado al ingresar a la universidad, la promoción (re-jerarquización) es un proceso voluntario al que se pueden adscribir los académicos del cuerpo académico regular, presentando sus antecedentes a la Comisión de Nombramientos y Promociones. La jerarquización y promoción considera dos áreas fundamentales, la formación del académico o postulante, y la obra realizada, la que incluye las siguientes dimensiones: docencia, investigación, creación (estudios y proyectos), creación artística, extensión, administración académica, experiencia profesional, compromiso institucional y relaciones interpersonales. (UTALCA, 2013)

Por otra parte, la calificación académica es "el acto por el cual la Universidad de Talca evalúa periódicamente la calidad del trabajo realizado por cada uno de sus académicos" (UTALCA, 2011, p.1). El propósito de la calificación es propender a la excelencia perfeccionando continuamente el desempeño académico. El proceso de calificación del cuerpo académico regular se realiza periódicamente para medir su desempeño, considerando en conjunto la calidad, responsabilidad y productividad con que el académico haya desempeñado las labores correspondientes a su jerarquía, y el cumplimiento del compromiso anual de desempeño, para lo cual se utilizan los conceptos de Bueno, Regular y Deficiente. (UTALCA, 2013).

La Calificación Académica se realiza por medio de dos instancias, los Comités de Calificación de Unidades Académicas (Facultad o Instituto) y la Comisión Superior de Calificación, cuya composición y funcionamiento está normado. El proceso de calificación, es clave ya que permite la renovación o eventual desvinculación de los académicos, según la calificación obtenida.

Tabla 1: Jerarquías Académicas y Procesos de Calificación en la Universidad de Talca

\begin{tabular}{|l|l|l|l|}
\hline Jerarquía Académica & Organismo Evaluador & Periodos de Calificación & Relación Contractual \\
\hline Profesor Titular & $\begin{array}{l}\text { Comisión Superior de } \\
\text { Calificación }\end{array}$ & $\begin{array}{l}\text { A los 2 años para primer } \\
\text { contrato o con calificación } \\
\text { regular, sino cada 4 años }\end{array}$ & $\begin{array}{l}\text { Primer contrato por 2 } \\
\text { años y luego Indefinido } \\
\text { (hasta edad de retiro) }\end{array}$ \\
\cline { 1 - 4 } Profesor Asociado & $\begin{array}{l}\text { Profesor Asistente (nombrados } \\
\text { hasta la edad de retiro) }\end{array}$ & Comité de Calificación \\
\cline { 1 - 3 } $\begin{array}{l}\text { Profesor Asistente (sin } \\
\text { retiro) }\end{array}$ & Anualmente & $\begin{array}{l}\text { Anual, hasta la tercera } \\
\text { renovación }\end{array}$ \\
\hline Instructor & & & \\
\hline
\end{tabular}

Los Comités de Calificación, están encargados de la calificación y recomendación de renovación de contrato de los profesores instructores y asistentes con contratos anuales; proceso que se realiza cada año, y en caso de obtener una calificación "Mala" o "Regular" por segunda vez, implica la desvinculación de la universidad. De la misma forma, la Comisión Superior de Calificación, está encargada de la calificación de profesores titulares, asociados y asistentes, cuyos contratos son hasta edad de retiro (o indefinidos), y de proponer su renovación de contrato, así como para los profesores asistentes cuyo nombramiento sea por cuarta vez. El proceso se realiza a los dos años, para el primer contrato o en caso de ser calificado como "Regular", y cada cuatro años para los evaluados en periodos anteriores con calificación "Bueno"; quienes son evaluados con calificación "Mala" o "Regular" por segunda vez, son desvinculados de la universidad. 


\section{MÉTODO Y MUESTRA}

Para el análisis del sistema de evaluación de desempeño académico en la Universidad de Talca, se realizó una investigación exploratoria de tipo cualitativa, cuyo trabajo de campo implicó recolección y sistematización de información institucional, y la aplicación de una entrevista en profundidad semiestructurada a 5 académicos de 3 jerarquías pertenecientes a la planta regular [1 Profesor Titular (PT); 1 Profesor Asociado (PA); y 3 Profesores Asistentes (PAS 1, 2 y 3)], que desempeñan o han ocupado roles claves a nivel directivo.

La investigación se realizó bajo un enfoque de análisis de teoría fundamentada, para lo cual se transcribieron las entrevistas y luego se identificaron códigos de datos que fueron organizados en dimensiones y categorías de análisis preestablecidas y consistentes con el diseño de las pautas de entrevista. El análisis se realizó haciendo referencia a citas textuales, donde las opiniones vertidas anónimamente, se presentan según los códigos señalados previamente.

\section{RESULTADOS Y DISCUSIÓN}

A partir del análisis de las entrevistas en profundidad, se establecieron las dimensiones y categorías de análisis que muestra la tabla 2.

Tabla 2: Dimensiones y Categorías de Análisis de las Entrevistas.

\begin{tabular}{|l|l|c|}
\hline \multicolumn{1}{|c|}{ Dimensiones } & \multicolumn{1}{|c|}{ Categorías de Análisis } & Recurrencia \\
\hline \multirow{4}{*}{$\begin{array}{l}\text { Componentes y Estructura del } \\
\text { sistema }\end{array}$} & Compromiso de Desempeño & 2 \\
\cline { 2 - 3 } & Calificación Académica & 4 \\
\cline { 2 - 3 } & Jerarquización Académica & 2 \\
\cline { 2 - 3 } & Proceso de Retroalimentación & 3 \\
\cline { 2 - 3 } & Comisión de Calificación & 2 \\
\cline { 2 - 3 } $\begin{array}{l}\text { Proceso de Evaluación de } \\
\text { Desempeño }\end{array}$ & Comisión de Jerarquización & 3 \\
\hline \multirow{5}{*}{\begin{tabular}{l} 
Evimensiones y Estándares de \\
\cline { 2 - 3 }
\end{tabular}} & Periodicidad de la Evaluación & 6 \\
\cline { 2 - 3 } & Pertinencia y Suficiencia & 2 \\
\cline { 2 - 3 } & Claridad de las Dimensiones & 2 \\
\cline { 2 - 3 } & Valoración (Ponderación) & 5 \\
\cline { 2 - 3 } & Claridad en los Estándares & 4 \\
\cline { 2 - 3 } & Definición de los Estándares & 2 \\
\hline Relevancia Estratégica & Alineación Estratégica & 6 \\
\cline { 2 - 3 } & Alineación entre Unidades Académicas y Comisiones de & 3 \\
\hline \multirow{5}{*}{\begin{tabular}{l} 
Evaluación \\
\cline { 2 - 3 }
\end{tabular}} & Eficacia del Sistema & 3 \\
\cline { 2 - 3 } & Eficiencia del Sistema & 3 \\
\cline { 2 - 3 } & Promoción y Desarrollo Académico & 3 \\
\cline { 2 - 3 } & Sistema de Incentivos & 3 \\
\cline { 2 - 3 } & Evaluación del Sistema de Evaluación & 3 \\
\hline
\end{tabular}

Los componentes del sistema de evaluación de desempeño son identificados con claridad por los entrevistados, debido a que son instancias que todos los académicos han vivido en algún momento, destacando entre sus componentes: Calificación académica, compromisos de desempeño, jerarquización, y retroalimentación.

Respecto del proceso de calificación se menciona que "hay una calificación que se hace cada cuatro años y en ese momento se miran todos los antecedentes de las evaluaciones anuales y se emite la calificación" (PA). Sin embargo, existen algunas observaciones sobre su implementación, "tenemos problemas para un buen funcionamiento de este sistema, una cosa es lo que está planeado, lo que está en los reglamentos y otra cosa es lo que realmente se realiza" (PA), refiriéndose a que muchas veces los compromisos de desempeño, satisfactoriamente evaluados, no llevan a necesariamente a una buena calificación. Frente a 
esto, otro académico enfatiza "si a mí me califican mal dentro de cuatro años, mi percepción es que las evaluaciones intermedias no son del todo profundas como para que retroalimenten mi accionar y yo llegue a los cuatro años sin problema" (PAS3).

En cuanto a la jerarquización académica, que no es parte de la evaluación de desempeño directamente puesto que es una condición de entrada y de continuidad, un académico expone: "la jerarquización está fundamentalmente dada por las distintas categorías que hay, tenemos profesores instructores, hay asistentes, asociados y titulares $y$ en base a la productividad y el aporte que uno haga puede subir de categoría" (PT).

Sobre la comisión de calificación, se señala que "la mayoría de las veces estas comisiones funcionan bien... aunque ha habido oportunidades en que no. Basta con que haya ocurrido una sola vez como para preocuparse de mejorar, no solamente el instrumento, sino que la manera en cómo el contenido de ese instrumento es evaluado por las personas que están nominadas en estas comisiones especiales" (PAS2). La relevancia que tiene la toma de decisiones de las comisiones de calificación, radica en el impacto que tienen sobre la estabilidad laboral de un académico, así como en la pérdida de profesionales competentes o la mantención de académicos que no tienen un desempeño adecuado.

Los académicos se refieren a la comisión de jerarquización, indicando que debería tener estándares precisos, ya que muchas veces la jerarquía en la que queda un académico depende de los criterios de los evaluadores, más que de sus méritos en comparación con otros académicos. Uno de ellos señala que "el estándar tiene que estar bien definido, tienen que ser profesores titulares con una buena producción en investigación, etc. ...en el fondo me parece que la universidad debería establecer o empezar a trabajar fuerte en este tema" (PT).

En cuanto a la retroalimentación, los entrevistados concuerdan en que no existe un proceso formal, mediante el cual se les informe sobre los aciertos y errores de su desempeño en los períodos que son evaluados, este es un punto crítico dentro de cualquier sistema de evaluación de desempeño, ya que es a través de ella, que se pueden establecer cambios de actitud y mejoras en el desempeño, sin retroalimentación, los procesos resultan ineficaces e ineficientes. Frente a esto, un académico hace un mea culpa indicando que no sólo es un problema de diseño, sino que también de cultura "no nos gusta la crítica... por tanto a veces, académicos que quedan calificados como Bueno... se molestan cuando le hacen alguna observación respecto a su desempeño... eso se toma prácticamente como un agravio...eso es una cuestión cultural..." (PA).

La evaluación de desempeño debe ser un proceso sistemático, por lo que resulta pertinente la realización de compromisos anuales de desempeño, donde se revisa el actuar de los académicos durante dicho periodo. Un entrevistado señala: "el compromiso de desempeño es un documento que tú firmas cada año, en dónde efectivamente consignas las tareas que vas a desempeñar durante ese período, fundamentalmente haciendo un desglose, respecto del tiempo que dedicas a lo que es, docencia a investigación, a extensión, o a gestión según sea la tarea a la cual tu estés asignada" (PAS2).

En cuanto a la calificación académica, cuyas evaluaciones son anuales, bianuales o cada cuatro años según la jerarquía y calificaciones obtenidas previamente (ver tabla 1), es necesario considerar el compromiso y la evaluación anual de desempeño como una entrada de información relevante en el proceso, los resultados de la investigación muestran que en la práctica esto no se cumple a cabalidad por lo que se desvirtúa la utilidad del compromiso de desempeño y su evaluación, debido al desfase y desarticulación que se observa.

Respecto de los criterios de evaluación, se aprecia una discordancia entre la normativa y los criterios de evaluación utilizados, muchos aspectos de los procesos de evaluación (tanto jerarquización como calificación) quedan sujetos a los criterios de las comisiones (las que varían de acuerdo a la unidad académica a la que pertenezcan), traduciéndose en evaluaciones dispares, lo que es percibido como una inequidad en el sistema. Al respecto, un académico señala: "el tema es que hay muchos aspectos que quedan sujetos a criterios... una de las demandas de la asociación de académicos es precisamente transparentar esos criterios, que no queden sujetos a las personas que aplican la normativa" (PAS2).

Con respecto a la pertinencia y suficiencia de las dimensiones de evaluación, aspectos que son evaluados dentro de los procesos de calificación y jerarquización, existen opiniones divididas, mientras algunos consideran que "las dimensiones son adecuadas, cubre todo lo que es posible realizar en la universidad" (PA), otro expone que "muchas veces se evalúa, sin tomar en cuenta los acuerdos que se han realizados con los decanos" (PT). Esto pone en evidencia una desarticulación entre los compromisos de desempeño y las dimensiones y criterios de evaluación de las comisiones. 
En cuanto a la claridad de las dimensiones de evaluación se señala que "no son muy conocidos porque siempre se evalúa como mínimo el desempeño académico de la docencia y por supuesto mide lo que es publicaciones e investigaciones" (PAS1), indicando que existe un supuesto basado en la experiencia, que los académicos deben ser evaluados en al menos dos dimensiones, que por lo general son docencia e investigación. También se indica que "lo único que sé es que si estoy en la planta regular como mínimo yo tengo que hacer algo más que docencia... a veces por razones de buen servicio, a uno le pueden decir usted este semestre solo hace docencia, esas cosas a la larga son mal vistas" (PAS3).

Respecto de la valoración asignada a cada dimensión de evaluación, los académicos entrevistados coinciden en que es un aspecto complejo, "se tiende a sobrevalorar, todo lo que es producto de la actividad de investigación y a subvalorar todo lo que es la actividad docente o de servicio a la comunidad, e incluso la actividad tecnológica" (PA). Algunos de ellos indican que estas valoraciones dependen de los criterios de las comisiones, y como las comisiones son diferentes en cada evaluación y para cada unidad académica, esto produce inequidades en el sistema de evaluación de desempeño académico.

Por otro lado los estándares de evaluación se relacionan con aquellas medidas que hacen que un académico sea calificado como bueno, regular o insuficiente. En cuanto a la definición de los estándares, los académicos entrevistados señalan que "en realidad se deja sujeto a la discreción de las comisiones, entonces se confía en el buen criterio de los integrantes" (PA).También se señala que el sistema deja mucha libertad a los criterios de las comisiones, lo que genera una percepción de inequidad en el sistema. Al respecto uno de ellos señala "los estándares tampoco son tan conocidos, porque he sabido de profesores que tienen cinco cursos al semestre y otros que tienen tres y otros que tienen dos" (PAS1), haciendo alusión a que los estándares de evaluación no están claros.

Por último, la relevancia estratégica del sistema se aprecia a partir de la alineación estratégica que se genera desde la vinculación de las diferentes actividades planificadas por los académicos (a través de sus CDAs), que se van enlazando a los objetivos de las unidades académicas (CDUs), y estos objetivos se adhieren a los objetivos estratégicos corporativos, en este aspecto todos los académicos entrevistados tuvieron algo que decir, desde su perspectiva, están de acuerdo con que existe alineación: "tienes un compromiso de desempeño académico que es individual pero supuestamente ese compromiso de desempeño tiene que estar ajustado a las metas y los objetivos estratégicos de tu unidad académica y esa unidad académica a su vez está comprometida con la institución, o sea la misión institucional es cumplida desde las bases a través de las unidades" (PAS2)

Sin embargo hay opiniones disimiles, donde un académico expresa que "en algunas áreas si se alinean y en otras no, yo creo aquí falta también ordenar un poco, porque en el fondo la pregunta es, ¿qué quiere hacer la universidad para el futuro?, hay contradicciones..." (PT). Al mismo tiempo, se enfatiza en la relevancia que tiene este sistema de evaluación para mejorar la calidad de los servicios de la universidad, "el sistema de evaluación de desempeño académico es bastante fundamental para la universidad, yo diría que es una de las claves para que la universidad funcione con calidad y tenga un buen desempeño como tal" (PA).

Respecto de la alineación de las unidades académicas con las comisiones de evaluación hay coincidencia en que es posible mejorar "... una cosa es lo que uno hace, otra cosa es lo que uno compromete y otra cosa es lo que se califica, hay tres elementos seguidos" (PAS1). Sobre ello, algunos entrevistados piensan que los compromisos de desempeño, visados por sus unidades no se vinculan totalmente a los resultados de las calificaciones emitidos por las comisiones de calificación, uno de ellos dice "...es un mal regalo para un profesor que el jefe le diga: te encargo esto y el profesor acepte (como le va a decir que no a su jefe) $y$ después otra comisión, que no es su jefe, lo evalúa mal" (PAS1). Lo anterior, evidencia la existencia de una debilidad dentro del sistema de evaluación, puesto que se percibe una incongruencia entre ambos componentes de la evaluación, las evaluaciones asociadas a los compromisos de desempeño y la evaluación de la comisión de calificación, lo que puede presentar riesgos para la confianza que entrega el sistema a los académicos.

En cuanto a la influencia que tiene el sistema de evaluación para mejorar la eficacia de la universidad, los académicos entrevistados coinciden en que el sistema es un aporte, uno de ellos dice que "contribuye a mejorar la eficacia, porque... hay metas que cumplir, nosotros no tenemos la obligación de cumplir horarios, pero si estamos obligados a cumplir las metas que comprometemos..." (PA). La influencia del sistema de evaluación para mejorar la eficiencia de la universidad, no es percibida claramente por los académicos, mientras algunos indican que el sistema aporta a esta propósito, otros señalan que el sistema no evalúa la eficiencia e incluso uno de ellos indica que "siento que tenemos titulares en la universidad que no están cumpliendo su trabajo y deberíamos exigirlo" (PT). 
Uno de los académicos entrevistados indica respecto a la promoción y desarrollo de carrera: "esos dos sistemas juegan por carriles separados (calificación y jerarquización) y no es tan malo que sea así porque una cosa no lleva a la otra, porque en la universidad no hay norma que a uno lo obligue a cambiar de jerarquía" (PAS3). En relación a los incentivos, hay opiniones divididas, mientras algunos indican que no existen, otros se refieren a un bono de treceavo sueldo en el caso de ser calificados como Bueno; uno de ellos lo describe "...un decimotercer sueldo que la universidad concede que está sujeto a tener una calificación buena, si una persona tiene calificación regular no tiene derecho...” (PA).

Sobre la evaluación del sistema, los académicos consultados indican que el sistema está en una mejora continua, sin embargo el monitoreo no es conocido en detalle, uno de ellos indica que "se está revisando permanentemente y también se ha sometido a la opinión de los pares que nos visitan en los procesos de acreditación, ellos también han tenido la posibilidad de emitir juicios respecto al sistema” (PA).

\section{CONCLUSIONES}

El análisis de las entrevistas realizadas, muestra que el sistema de evaluación de desempeño académico de la Universidad de Talca, es percibido como relevante y necesario para alcanzar mayor eficacia y eficiencia a nivel institucional, destacando las siguientes fortalezas en el sistema: i) El sistema cuenta con una alta validación y sus procesos se encuentran internalizados por los académicos; ii) Los componentes y la estructura del sistema operan con claridad y regularidad; iii) Se percibe que el sistema está consolidado y presenta mejoras sistemáticas a través del tiempo; y iv) El sistema es periódicamente revisado, siendo adaptado a los cambios institucionales y del sistema universitario. Los entrevistados destacan que la universidad cuenta con un sistema que es conocido, aplicado y valorado por sus académicos, lo que contribuye a mejorar sistemáticamente los procesos y facilita el logro de los objetivos institucionales.

Al mismo tiempo, se debe considerar que todo sistema de evaluación de desempeño es susceptible de mejora, es por ello que a partir del análisis realizado, se han identificado algunas brechas que permitirían optimizar el funcionamiento del sistema: i) Se puede lograr una mayor alineación entre los instrumentos y procesos que conforman el sistema, como son los compromisos de desempeño y la calificación académica, o una mayor vinculación entre esta última y la jerarquización; ii) La definición de estándares de evaluación más precisos, permitiría disminuir los niveles de ambigüedad y la posibilidad de aplicación de disparidad de criterios al realizar las evaluaciones; iii) El compromiso de desempeño académico se percibe como poco relevante para la calificación y jerarquización, por lo que dicho instrumento debiera ser vinculante con los procesos señalados; iv) El sistema de jerarquización no se visualiza plenamente convergente con los resultados obtenidos en la calificación académica; v) Se le atribuye una importancia relativa a las dimensiones de evaluación, lo que no se condice con las políticas y objetivos estratégicos de la universidad y sus unidades académicas. Igualmente, se da una importancia excesiva a la investigación y la docencia, desplazando a otras actividades relevantes para el alcance de los objetivos institucionales; y vi) No se percibe una retroalimentación clara respecto de los resultados obtenidos, lo que dificulta el mejoramiento y reorientación del desempeño de los académicos.

Finalmente, se destaca que el sistema es valorado por los entrevistados, por su alta importancia estratégica para la universidad, contando con un diseño adecuado, sustentado en estructuras y procesos que se encuentran validados, siendo necesario realizar mejoras de naturaleza táctica y operativa para fortalecer su eficacia y eficiencia, incrementando el capital simbólico que el sistema de evaluación de desempeño tiene como parte de la cultura de la Universidad de Talca.

\section{REFERENCIAS}

Alles, M., Desempeño por Competencias. Buenos Aires: Granica, Argentina (2005)

Altbach, P., Reisberg, L. y Rumbley, L. Trends in Global Higher Education: Tracking an Academic Revolution. UNESCO World Conference on Higher Education (2009)

Bernasconi, A., La profesionalización de la academia en Chile. Revista Calidad en la Educación No 28, Consejo Nacional de Educación, Chile (2008)

Bernasconi, A. La apoteosis del investigador y la institucionalización de la profesión académica. Estudios sobre Educación, VOL. 19, pp.139-163, España, (2010)

Berrios, P., Normas y percepciones sobre carrera académica en chile. Revista Calidad en la Educación No 28, Consejo Nacional de Educación, Chile (2008a) 
Berrios, P., Carrera académica: análisis empírico de su estructura y organización en Chile. Revista Calidad en la Educación No 29, Consejo Nacional de Educación, Chile (2008b)

Bohlander, G., Snell, S. y Sherman, A. W. Administración de Recursos Humanos. México: International Thomson Editores (2001)

Brunner, J. La idea de la Universidad pública en América Latina: Narraciones en escenarios divergentes.

Educación XX1, 17 (2), 17-34. DOI: 10.5944/educxx1.17.2.11477 (2014)

Cavalli, A., y Moscati, R. Academic systems and professional conditions in five European countries. European Review, 18(1), 35-53 (2010)

Chiavenato, I. Administración de Recursos Humanos. Bogotá, Colombia: Mc Graw Hill Interamericana, S.A. (2000)

Chiavenato, I. Gestión del Talento Humano. Ciudad de México: Mc Graw Hill, (2009)

CINDA Evaluación de Desempeño Docente y Calidad de la Docencia Universitaria. Alfabeta Impresores. Santiago de Chile (2008)

Espinoza, O. y González, L., Evaluación de desempeño en la educación superior: un modelo de análisis. En Aedo, A. (Ed.) Desafíos y perspectivas de la dirección estratégica de las instituciones universitarias. Ediciones CNA-Chile (2009)

Fernández, E., Compromisos de desempeño, incentivos y evaluación del trabajo académico. Revista Calidad en la Educación No 28, Consejo Nacional de Educación, Chile (2008)

Fernández Lamarra, N. y Coppola, N., Evaluación de la docencia universitaria desde un abordaje institucional. Revista Iberoamericana de Evaluación Educativa, Volumen 3, Número 1e (2010)

González, S.; Brunner, J.J., y Salmi, J. Comparación Internacional de Remuneraciones Académicas: Un estudio exploratorio. Revista Calidad en la Educación No 39, Consejo Nacional de Educación, Chile (2013)

Gil, J., La evaluación de competencias laborales. Educación XX1. 10, pp. 83-106, España (2007)

Hatum, A., Alineando la Organización. Buenos Aires: Granica S.A. Argentina (2009)

Hitt, M.; Ireland, R., y Hoskisson, R. Administración Estratégica: Competitividad y Conceptos de Globalización. Editorial Thomson, México. (2004)

Huerta, L. y González, P., Sistema de Evaluación del Desempeño Académico en la Universidad de Talca. Presentación del Taller de Gestión por resultados: Los convenios de desempeño 22 y 23 de Junio de 2010, Santiago. http://www.cpce.cl/anillo/59-taller-gestion-santiago-20101, Acceso: 14/12/2013.

Marin, R.; Guzman, I.; Márquez, A. y Pena, M., La evaluación de competencias docentes en el modelo DECA: anclajes teóricos. Formación Universitaria., vol.6, n.6, pp. 41-54, Chile (2013)

Macfarlane, B., The Morphing of Academic Practice: Unbundling and the Rise of the Para-academich, Higher Education Quarterly, Volume 65, No. 1, January, pp 59-73, (2011)

Rocha, R., Escala de opinión de los estudiantes sobre la efectividad de la docencia (EOEED) en educación superior. Formación Universitaria, vol.6, n.6, pp. 13-22, Chile (2013).

Rojas, A. Programa de Gobierno Universitario: Periodo 2014-2018. Universidad de Talca, Chile http://www.utalca.cl/link.cgi//GobiernoU/1938, Acceso: 15/07/2014. (2014)

Rueda, M., La evaluación del desempeño docente en las universidades públicas de México. Revista Iberoamericana de Evaluación Educativa, http://www.rinace.net/riee/vol1-num3_e/art1.pdf, Acceso: 20/10/2012. (2008a)

Rueda, M., La evaluación del desempeño docente en la universidad. Revista Electrónica de Investigación Educativa, vol.10, , http://www.scielo.org.mx/scielo.php?script=sci_arttext\&pid=S1607-

40412008000300002\&lng=es\&nrm=iso, Acceso: 15/12/2014 (2008b)

Salmi, J. El desafío de crear universidades de rango mundial. Banco Mundial en coedición con Mayol Ediciones S.A. Colombia, (2009) 
UTALCA. Resolución Universitaria N¹348: Promulga Acuerdo modifica el reglamento de funcionamiento de los comités y comisión superior de calificaciones y fija texto refundido. Universidad de Talca, Chile, http://transparencia.utalca.cl/index.php, Acceso: 18/07/2014. (2011)

UTALCA. Resolución Universitaria N¹843 Promulga acuerdo que modifica la Ordenanza General del Académico y fija texto refundido, Universidad de Talca, Chile, http://transparencia.utalca.cl/index.php, Acceso: 18/07/2014. (2013)

Vain, P.D. La Evaluación de la Docencia Universitaria: Un problema complejo. Serie Estudio N4, CONEAU Ministerio de Educación Argentina, http://eco.mdp.edu.ar/cendocu/repositorio/00207.pdf, Acceso: 13/12/2011 (1998) 